\title{
Inter-scan reproducibility of coronary calcium measurement using Multi Detector-Row Computed Tomography (MDCT)
}

\author{
Siamak Sabour · A. Rutten · Y. T. van der Schouw • \\ F. Atsma · D. E. Grobbee · W. P. Mali · M. E. L. Bartelink · \\ M. L. Bots · M. Prokop
}

Received: 8 September 2006/ Accepted: 12 March 2007/Published online: 11 April 2007

(C) Springer Science+Business Media B.V. 2007

\begin{abstract}
Purpose To assess inter-scan reproducibility of coronary calcium measurements obtained from Multi Detector-Row CT (MDCT) images and to evaluate whether this reproducibility is affected by different measurement protocols, slice thickness, cardiovascular risk factors and/or technical variables.

Design Cross-sectional study with repeated measurements.

Materials and methods The study population comprised 76 healthy women. Coronary calcium was assessed in these women twice in one session using 16-MDCT (Philips Mx 8000 IDT 16). Images were reconstructed with $1.5 \mathrm{~mm}$ slice thickness and $3.0 \mathrm{~mm}$ slice thickness. The 76 repeated scans were scored. The Agatston score, a volume measurement and a mass measurement were assessed. Reproducibility was determined by estimation of mean, absolute, relative difference, the weighted kappa value for agreement and the Intra-class correlation coefficient (ICCC).

Results Fifty-five participants $(72.4 \%)$ had a coronary calcification of more than zero in Agatston (1.5 mm slice thickness). The reproducibility of coronary calcium
\end{abstract}

S. Sabour - Y. T. van der Schouw $\cdot$ F. Atsma .

D. E. Grobbee · M. E. L. Bartelink · M. L. Bots ( $\square)$

Julius Center for Health Sciences and Primary Care, University

Medical Center Utrecht, Str. 6.131, Heidelberglaan 100, 3584

CX Utrecht, The Netherlands

e-mail: m.1.bots@umcutrecht.nl

A. Rutten · W. P. Mali · M. Prokop

Radiology Department, University Medical Center Utrecht,

Utrecht, The Netherlands

S. Sabour

Ministry of Health and Medical Education, Tabriz University of Medical Sciences, Tabriz, I.R. Iran measurements between scans in terms of ranking was excellent with Intra-class correlation coefficients of $>0.98$, and kappa values above 0.80 . The absolute difference in calcium score between scans increased with increasing calcium levels, indicating that measurement error increases with increasing calcium levels. However, no relation was found between the mean difference in scores and calcium levels, indicating that the increase in measurement error is likely to result in random misclassification in calcium score. Reproducibility results were similar for $1.5 \mathrm{~mm}$ slices and for $3.0 \mathrm{~mm}$ slices, and equal for Agatston, volume and mass measurements.

Conclusion Inter-scan reproducibilility of measurement of coronary calcium using images from MDCT is excellent, irrespective of slice thickness and type of calcium parameter.

Keywords Multi Detector-Row CT (MDCT) · Coronary artery calcification - Atherosclerosis · Epidemiology · Reproducibility

\section{Introduction}

A considerable proportion of the western society is at risk of suffering a cardiovascular event during life. Atherosclerosis is one of the main underlying processes. Noninvasive assessment of atherosclerosis is important since it allows studies into the etiology and consequences of early and advanced atherosclerosis in populations at large [1]. The last two decades, measurement of coronary artery calcification (CAC) using computer tomography (CT) has been used to assess coronary atherosclerosis non-invasively. The presence, and more importantly, the quantity of $\mathrm{CAC}$, relates well with the overall severity of the athero- 
sclerotic process [2]. Several studies have demonstrated a strong relation between coronary calcium burden and the incidence of myocardial infarction, a relation which was independent of age [3, 4].

Most of the evidence on determinants and consequences of coronary calcium is based on data obtained with electron beam CT (EBCT) [5-7]. The availability of EBCT scanners is modest, whereas the Multi Detector-Row CT (MDCT) scanners are more widely available and also allow for detection of coronary calcium. Current data suggest that EBCT and MDCT give comparable results [8, 9]. In contrast to EBCT, however, data on reproducibility of CAC measurements using MDCT images is not widely available $[10,11]$, but information is relevant. Furthermore, due to technical improvement, slice thickness of the images has become smaller which may affect the likelihood of detecting coronary calcium, and hence its reproducibility.

We set out to study inter-scan reproducibility of coronary calcium measurements from MDCT images and to evaluate whether reproducibility is affected by different measurement protocols, slice thickness, selected cardiovascular risk factors and technical variables.

\section{Materials and methods}

Participants were recruited from the PROSPECT study [12], cohort of 17,357 healthy breast-cancer screening participants, aged 49-70 years, living in Utrecht and surroundings, enrolled between 1993 and 1997. Between October 2002 and December 2004, a random selection of 1,996 women were invited by mail and 1,000 (50.1\%) who were postmenopausal and did not use contraceptives or hormone replacement therapy answered positively. Of these 1,000 women, a random selection of 573 underwent a MDCT examination during a single visit and 76 of them were scanned twice. The Medical Ethical Committee of the University Medical Center Utrecht approved the study and written informed consent was obtained from all participants.

Current cardiovascular drug use (blood pressure lowering, lipid lowering and glucose lowering drugs) was assessed by asking women to bring all packages to the study centre. Smoking behavior, medical history and cardiovascular family history were assessed by a questionnaire. Height and weight were measured and body mass index was calculated as weight divided by height squared $(\mathrm{kg} /$ $\mathrm{m}^{2}$ ). Waist-to-hip ratio (WHR) was assessed. Systolic and diastolic blood pressures were measured at both arms with an automated and calibrated blood pressure device (DINAMAP $^{\mathrm{TM}} \mathrm{XL}$, Critikon, Johnson \& Johnson, Tampa, Florida, USA) with the subject in supine position. A venous blood sample was drawn after an overnight fast of at least eight hours. Plasma total cholesterol, plasma triglycerides, and plasma glucose were measured using standard enzymatic procedures. High-density lipoprotein (HDL) cholesterol was measured by the direct method (inhibition, enzymatic). Low-density lipoprotein (LDL) cholesterol was calculated using the Friedewald formula.

\section{Coronary imaging and calcium measurements}

The amount of calcium in the coronary arteries was assessed with a Multi Detector-Row CT (MDCT) scanner (Mx 8000 IDT 16, Philips Medical Systems, Best, The Netherlands). Subjects were positioned within the gantry of the MDCT scanner in supine position. During a single breath hold, images of the heart, from the level of the tracheal bifurcation to below the base of the heart, were acquired using prospective ECG triggering at 50-80\% of the RR-interval, depending on the heart rate. Scan parameters were $16 \times 1.5 \mathrm{~mm}$ collimation, $205 \mathrm{~mm}$ field of view (FOV), $0.42 \mathrm{~s}$ rotation time, $0.28 \mathrm{~s}$ scan time per table position, $120 \mathrm{kVp}$ and $40-70 \mathrm{mAs}$ (patient weight $<70 \mathrm{~kg}$ : 40 mAs; 70-90 kg: 55 mAs; >90 kg: 70 mAs). Scan duration was approximately $10 \mathrm{~s}$, depending on heart rate and patient size. We had the participant get up from the table and lay down again since in studies on change in CAC over one year it is not realistic to assume exactly the same position of the participant at both occasions. Therefore our patients sat up and consequently moved slightly between scans to mimic two separate scan runs.

From the acquired raw data, the whole volume was reconstructed with an intermediate reconstruction algorithm in non-overlapping data sets of $1.5 \mathrm{~mm}$ and $3 \mathrm{~mm}$ thick sections. Quantification of coronary calcium was performed on a separate workstation with software for calcium scoring (Heartbeat-CS, EBW, Philips Medical Systems, Best, The Netherlands). All regions with a density over 130 Hounsfield units were identified as potential calcifications.

After completing a training-program, one scan reader (AR) who was unaware of the scores of the first scan, manually selected the calcifications within one of the coronary arteries (left main, left anterior descending, left circumflex, right coronary artery, and PDA) and scored the second scan of the participants. To reduce the influence of noise, the minimum size of a calcified lesion was set at $0.5 \mathrm{~mm}^{2}$. The peak density in Hounsfield units and the area in $\mathrm{mm}^{2}$ of each selected region were calculated. The Agatston [13] calcium score was obtained by multiplying the area by a weighting factor that is dependent on the peak signal anywhere in the lesion. The scores of individual lesions were added to obtain the Agatston calcium score for the entire coronary tree. The total cal- 
cium volume was calculated by multiplying the area of the calcified lesion (measured in square millimeters) by section thickness $(1.5 \mathrm{~mm}$ and $3.0 \mathrm{~mm})$. The calcium volume for each coronary vessel was computed by summing the volumes of the lesions in that vessel for all sections. Finally, the total volume from all the vessels became the calcium volume for a subject. The mass method uses volumetric, density information and a calibration phantom of hydroxyapatite to calculate the actual mass of the calcified plaques [14].

In addition, information on breathing artifact (inconsistency of sternum bone in sagital section in $\mathrm{mm}$ ), noise (standard deviation of enhancement in fixed cardiac area of $212 \mathrm{~mm}^{2}$ ) and mean heart rate (beats/min) during scan acquisition was collected.

\section{Data analysis}

The mean and standard deviations (SD) of coronary calcium were calculated for all scoring methods separately. Because of the skewed distribution of scores, medians were also computed. The Intra-class correlation coefficient was estimated for between scans data and for 1.5 and $3.0 \mathrm{~mm}$ slices thicknesses separately. The mean difference in score between scans was calculated as well as the absolute and relative differences.

To distinguish between random differences or systematic difference, information on mean and absolute differences is needed. One may assume a priori a nondifferential misclassification in the calcium scores, but one has to show that with the results. When the chance of the 2nd result being higher or lower is equal, one would expect a mean difference of zero, with some standard deviation. The absolute difference will not be zero since all differences are 'absolutised', but it is expected that at least the mean difference is much less than the absolute difference. If however the chance of a higher or lower value in the 2nd scan is not equal, the mean difference will be plus or minus a certain value. In addition, the absolute difference will have a value close to that of the mean difference. Therefore we need both parameters.

To estimate a weighted kappa as measure of agreement of categorical variables, subjects were divided into four groups according to the mean Agatston score as proposed by Rumberger et al. [15]: A: 0-9 (absent-minimal), B: 1099 (mild), C: 100-399 (moderate) and D: (400 (severe degree of calcification). This categorization is specifically for the calcium scoring method according to Agatston. Therefore we additionally categorized all scoring methods in their quartiles to calculate kappa as measure of agreement for all scoring methods.
The relation between risk factors, technical variables and measurement error was assessed using Spearman correlation coefficients. In a similar manner the relation between calcium level and measurement error was examined. Since logarithms of coronary calcium scores have generally been used in statistical analyses in other papers, we also studied the reproducibility of logarithmic transformed calcium score. Logarithmic analysis of coronary calcium scores was performed by calculating natural $\log$ of coronary calcium scores +0.001 (ln $(\mathrm{CCS}+0.001))$ because the logarithm of coronary calcium scores alone excludes all subjects with zero scores [16]. We defined relative difference as absolute difference divided by the mean calcium level multiplied in 100 and expressed in percent. Data analysis was performed with SPSS for windows, version 12.0. A statistically significant difference was assumed when the two-sided $P$-value was less than 0.05 .

\section{Results}

Mean age was $67.3 \pm 5.2$ years. Fifty-five participants (72.4\%) had a coronary calcification more than zero in Agatston (1.5 mm slice thickness). Table 1 shows the general characteristics of the 76 women who had two MDCT scans.

Table 2 presents information on calcium distributions by various scoring techniques and reproducibility results, by slice thickness. Overall, calcium scores were higher when based on the $1.5 \mathrm{~mm}$ slice thickness than based on the $3.0 \mathrm{~mm}$ slice thicknesses. The kappa agreement and Intra-class correlation coefficients between the two scans were high for all scoring methods, indicating that with respect to ranking of subjects all three methods are doing well. In addition, the mean differences in scores were relatively small compared to the absolute differences for all measurements, suggestion no systematic measurement errors. Finally, results for the scans with $1.5 \mathrm{~mm}$ slice thickness were similar to those for the $3.0 \mathrm{~mm}$ slice thickness (Table 2).

Table 3 presents the relation of cardiovascular risk factors with inter-scan mean difference. No consistent relations were found between risk factor levels and measurement error. Importantly, however was the observation that calcium level or the logarithm of the coronary calcium scores were not related to the mean difference between scans, whereas they were significantly related to the absolute and relative differences (Table 4, Figs. 1 and 2). These observations suggest that measurement error increases with increasing CAC levels, yet that this occurs in a random way. 
Table 1 Characteristics of studied population $(N=76)$

\begin{tabular}{lcc}
\hline & Mean & Std. deviation \\
\hline Age (year) & 67.3 & 5.2 \\
BMI $\left(\mathrm{Kg} / \mathrm{m}^{2}\right)$ & 26.3 & 3.9 \\
WHR & 0.84 & 0.06 \\
SBP (mmHg) & 133.9 & 18.9 \\
DBP (mmHg) & 71.7 & 9.1 \\
Total cholesterol (mmol/l) & 6.09 & 0.86 \\
LDL cholesterol (mmol/1) & 4.31 & 0.97 \\
HDL cholesterol (mmol/l) & 1.51 & 0.36 \\
Triglycerides (mmol/l) & 1.28 & 0.62 \\
Glucose (mmol/l) & 4.05 & 0.69 \\
Heart rate (beat/minute) & 72 & 11 \\
Current smoking (\%)* & 11 & \\
Former smoking (\%) & 43 & \\
Previous CVD (\%) & 1 & \\
Family history of CAD in either parents $(\%)$ & 10 & \\
\hline
\end{tabular}

$\mathrm{BMI}=$ Body $\quad$ Mass $\quad$ Index $; \quad$ CAD $=$ Coronary Artery Diseases; DBP $=$ Diastolic Blood Pressure LDL = Low Density Lipoprotein; HDL = High Density Lipoprotein; SBP = Systolic Blood Pressure; WHR = Waist to Hip Ratio

* Percentages have been rounded

\section{Discussion}

With respect to ranking of subjects, the inter-scan reproducibility of coronary calcium measurements by MDCT using Agatston, volume and mass scoring algorithms is excellent. The inter-scan reproducibility showed no major differences between scoring methods. The slice thickness did not affect reproducibility, nor did heart rate and technical parameters. Measurement error was related to increased coronary artery calcification, although our findings suggest that the error in the measurements is a random phenomenon.

Our findings, i.e., no major differences between scoring methods are in contrast with several reports on reproducibility based on EBCT scanning. Direct comparison of the findings of these studies with those of other is difficult since the parameters used to indicate reproducibility differ between studies. Furthermore, potentially the prevalence of $\mathrm{CAC}$ and its extent may affect reproducibility, as our findings suggest that measurement error increases with increasing CAC levels. Also the sizes of the studies differ which have undeniable effects on reproducibility results. However, our results are similar to those of by Rumberger and Kaufman [17], who compared these three methods and did not find any one method preferable to another in terms

Table 2 Characteristics of different coronary calcium scoring methods; effect of slice thickness on inter-scan reproducibility

\begin{tabular}{|c|c|c|c|c|c|c|}
\hline & $\begin{array}{l}\text { Mass 1st } \\
\text { Scan }\end{array}$ & $\begin{array}{l}\text { Mass 2nd } \\
\text { Scan }\end{array}$ & $\begin{array}{l}\text { Volume 1st } \\
\text { Scan }\end{array}$ & $\begin{array}{l}\text { Volume 2nd } \\
\text { Scan }\end{array}$ & $\begin{array}{l}\text { Agatston 1st } \\
\text { Scan }\end{array}$ & $\begin{array}{l}\text { Agatston 2nd } \\
\text { Scan }\end{array}$ \\
\hline \multicolumn{7}{|l|}{ Slice thickness $1.5 \mathrm{~mm}$} \\
\hline Mean & 32.21 & 31.88 & 154.52 & 149.40 & 170.33 & 163.63 \\
\hline Median & 6.15 & 6.05 & 39.97 & 36.52 & 31.85 & 32.00 \\
\hline $\begin{array}{l}\text { Agreement (k) Rumberger } \\
\text { categories }\end{array}$ & 0.97 & & 0.89 & & 0.87 & \\
\hline Agreement (k) Quartiles & 0.84 & & 0.81 & & 0.88 & \\
\hline Mean difference & 0.3 & & 5.1 & & 6.7 & \\
\hline Absolute difference & 4.0 & & 22.3 & & 24.3 & \\
\hline Relative difference $(\%)$ & 12.4 & & 14.6 & & 14.5 & \\
\hline ICCC* & 0.99 & & 0.99 & & 0.98 & \\
\hline \multicolumn{7}{|l|}{ Slice thickness $3.0 \mathrm{~mm}$} \\
\hline Mean & 25.57 & 25.45 & 131.45 & 126.98 & 140.06 & 135.82 \\
\hline Median & 4.00 & 3.65 & 30.30 & 21.90 & 20.30 & 18.00 \\
\hline $\begin{array}{l}\text { Agreement (k) Rumberger } \\
\text { categories }\end{array}$ & 0.92 & & 0.83 & & 0.73 & \\
\hline Agreement (k) Quartiles & 0.84 & & 0.84 & & 0.84 & \\
\hline Mean difference & 0.1 & & 4.4 & & 4.2 & \\
\hline Absolute difference & 3.5 & & 18.7 & & 21.3 & \\
\hline Relative difference $(\%)$ & 13.7 & & 14.7 & & 15.4 & \\
\hline ICCC $*$ & 0.99 & & 0.98 & & 0.98 & \\
\hline
\end{tabular}

* Intra-class correlation coefficient 
Table 3 Relationship between cardiovascular risk factors and inter-scan mean difference of coronary calcium scoring methods by MDCT (Slice thickness $1.5 \mathrm{~mm}$ )

\begin{tabular}{|c|c|c|c|c|c|c|}
\hline \multirow{3}{*}{$\frac{\text { CCS methods }}{\text { Biological variables }}$} & \multicolumn{6}{|c|}{ Inter-scan mean difference } \\
\hline & \multicolumn{2}{|l|}{ Mass } & \multicolumn{2}{|l|}{ Volume } & \multicolumn{2}{|c|}{ Agatston } \\
\hline & $\mathrm{r}$ & $P$-value & $\mathrm{r}$ & $P$-value & $\mathrm{r}$ & $P$-value \\
\hline BMI $\left(\mathrm{Kg} / \mathrm{m}^{2}\right)$ & 0.04 & 0.73 & 0.03 & 0.74 & 0.02 & 0.80 \\
\hline Age (year) & 0.18 & 0.10 & 0.31 & 0.00 & 0.28 & 0.01 \\
\hline Smoking(Categorical) & -0.00 & 0.98 & 0.04 & 0.71 & 0.07 & 0.49 \\
\hline WHR & -0.03 & 0.73 & 0.08 & 0.48 & 0.13 & 0.24 \\
\hline SBP (mmHg) & 0.10 & 0.37 & 0.16 & 0.14 & 0.24 & 0.03 \\
\hline $\mathrm{DBP}(\mathrm{mmHg})$ & 0.16 & 0.14 & 0.05 & 0.61 & 0.11 & 0.34 \\
\hline Cholesterol (mmol/l) & -0.27 & 0.05 & -0.12 & 0.40 & -0.20 & 0.17 \\
\hline $\mathrm{LDL}(\mathrm{mmol} / \mathrm{l})$ & -0.18 & 0.10 & -0.19 & 0.09 & -0.09 & 0.40 \\
\hline HDL (mmol/l) & -0.04 & 0.72 & -0.16 & 0.14 & -0.11 & 0.34 \\
\hline Triglyceride (mmol/l) & -0.02 & 0.85 & 0.13 & 0.24 & 0.11 & 0.34 \\
\hline Glucose (mmol/l) & 0.16 & 0.24 & -0.00 & 0.98 & 0.00 & 0.98 \\
\hline Mean heart rate & -0.03 & 0.77 & -0.03 & 0.73 & -0.02 & 0.81 \\
\hline \multicolumn{7}{|l|}{ Technical variables } \\
\hline Mean breathing artifact & 0.01 & 0.88 & -0.03 & 0.78 & -0.02 & 0.87 \\
\hline Mean SD of noise & 0.13 & 0.26 & 0.08 & 0.49 & 0.07 & 0.52 \\
\hline \multicolumn{7}{|l|}{ Coronary calcium } \\
\hline Mean mass score & 0.00 & 0.98 & & & & \\
\hline Mean volume score & & & 0.03 & 0.75 & & \\
\hline Mean Agatston score & & & & & 0.02 & 0.86 \\
\hline Mean log mass score & 0.00 & 0.99 & & & & \\
\hline Mean log volume score & & & 0.03 & 0.76 & & \\
\hline Mean log Agatston score & & & & & 0.02 & 0.85 \\
\hline
\end{tabular}

BMI = Body Mass Index; DBP = Diastolic Blood Pressure; LDL = Low Density Lipoprotein; HDL = High Density Lipoprotein; $r$ = spearman correlation coefficient; SBP = Systolic Blood Pressure; WHR = Waist to Hip Ratio

of reproducibility of results from consecutive scans in a patient.

Although the correlation between inter-scan measurements is excellent [18, 19], it still occurs that subjects with small deposits of calcium in scan one may have larger deposits of calcium in the 2nd scan, which leads to proportionally larger error in reproducibility. This has triggered other studies [20] on reproducibility to suggest that "the variability is partially a function of the absolute calcium score and inversely related to it', implicating that low coronary calcium scores may not be reproducible. However, our results could not confirm this.

Besides different algorithms for calcium scoring, slice thickness has been reported to affect the reproducibility of scoring protocols. In our study, the reproducibility of the coronary calcium measurements by MDCT was similar for $1.5 \mathrm{~mm}$ as for $3.0 \mathrm{~mm}$ slice thickness, and equal for Agatston, volume and mass measurements confirming the results by Rumberger and Kaufman [17].
The implications of our main findings depend on the research question that is asked in studies using CAC measurements. When the interest is using CAC measurements for prognostic studies our results for kappa and ICCC show that ranking of subjects is adequate based on one CT scan. So the need for duplicate CAC scan is absent. The fact that measurement error increases with increasing CAC values, is in prognostic studies not of major importance since the categorization of individuals seems adequate. When the interest is in etiologic studies using CAC as outcome parameter, our findings show that risk factor relations will be validly estimated since none of the risk factors relates to measurement error. When the interest is in using CAC as risk factor for future events (assessment of relative risks), it is most likely that in analyses with CAC as continues variable the magnitude of association of high CAC levels with events reflects an underestimation of the true magnitude. The direction of the relation will not change since based on our results measurement error is random, leading to random misclassification of the exposure variable. When the interest is in diagnostic value of 
Table 4 Relationship between cardiovascular risk factors and inter-scan absolute and relative difference of coronary calcium scoring methods by MDCT (Slice thickness $1.5 \mathrm{~mm}$ )

\begin{tabular}{|c|c|c|c|c|c|c|}
\hline \multirow{3}{*}{$\frac{\text { CCS methods }}{\text { Biological variables }}$} & \multicolumn{6}{|c|}{ Inter-scan relative difference } \\
\hline & \multicolumn{2}{|l|}{ Mass } & \multicolumn{2}{|c|}{ Volume } & \multicolumn{2}{|c|}{ Agatston } \\
\hline & $\mathrm{r}$ & $P$-value & $\mathrm{r}$ & $P$-value & $\mathrm{r}$ & $P$-value \\
\hline $\mathrm{BMI}\left(\mathrm{Kg} / \mathrm{m}^{2}\right)$ & 0.07 & 0.53 & 0.08 & 0.46 & 0.09 & 0.43 \\
\hline Age (year) & 0.21 & 0.06 & 0.24 & 0.03 & 0.117 & 0.12 \\
\hline Smoking(Categorical) & -0.03 & 0.73 & -0.07 & 0.51 & -0.14 & 0.20 \\
\hline WHR & 0.07 & 0.55 & 0.05 & 0.66 & 0.05 & 0.66 \\
\hline SBP $(\mathrm{mmHg})$ & 0.06 & 0.57 & 0.04 & 0.68 & 0.11 & 0.32 \\
\hline DBP (mmHg) & 0.32 & 0.004 & 0.31 & 0.005 & 0.33 & 0.003 \\
\hline Cholesterol (mmol/l) & 0.13 & 0.37 & 0.10 & 0.50 & 0.00 & 1.00 \\
\hline $\mathrm{LDL}(\mathrm{mmol} / \mathrm{l})$ & -0.14 & 0.21 & -0.17 & 0.12 & -0.18 & 0.11 \\
\hline HDL (mmol/l) & 0.07 & 0.52 & 0.04 & 0.67 & 0.06 & 0.57 \\
\hline Triglyceride (mmol/l) & 0.03 & 0.78 & 0.07 & 0.49 & 0.00 & 0.99 \\
\hline Glucose $(\mathrm{mmol} / \mathrm{l})$ & 0.23 & 0.09 & 0.26 & 0.05 & 0.24 & 0.08 \\
\hline Mean heart rate & -0.01 & 0.91 & 0.01 & 0.93 & 0.00 & 0.97 \\
\hline \multicolumn{7}{|l|}{ Technical variables } \\
\hline Mean breathing artifact & 0.10 & 0.44 & 0.09 & 0.49 & 0.15 & 0.23 \\
\hline Mean SD of noise & 0.19 & 0.09 & 0.19 & 0.09 & 0.18 & 0.11 \\
\hline \multicolumn{7}{|l|}{ Coronary calcium } \\
\hline Mean mass score & 0.29 & 0.009 & & & & \\
\hline Mean volume score & & & 0.33 & 0.003 & & \\
\hline Mean Agatston score & & & & & 0.38 & 0.001 \\
\hline Mean log mass score & 0.29 & 0.010 & & & & \\
\hline Mean log volume score & & & 0.33 & 0.003 & & \\
\hline \multirow[t]{2}{*}{ Mean log Agatston score } & & & & & 0.37 & 0.001 \\
\hline & \multicolumn{6}{|c|}{ Inter-scan absolute difference } \\
\hline \multicolumn{7}{|l|}{ Technical variables } \\
\hline Mean breathing artifact & 0.12 & 0.32 & 0.12 & 0.33 & 0.15 & 0.22 \\
\hline Mean SD of noise & 0.20 & 0.08 & 0.19 & 0.09 & 0.15 & 0.17 \\
\hline \multicolumn{7}{|l|}{ Coronary calcium } \\
\hline Mean mass score & 0.86 & $<0.001$ & & & & \\
\hline Mean volume score & & & 0.84 & $<0.001$ & & \\
\hline Mean Agatston score & & & & & 0.89 & $<0.001$ \\
\hline Mean log mass score & 0.86 & $<0.001$ & & & & \\
\hline Mean log volume score & & & 0.83 & $<0.001$ & & \\
\hline Mean log Agatston score & & & & & 0.89 & $<0.001$ \\
\hline
\end{tabular}

BMI = Body Mass Index; DBP = Diastolic Blood Pressure; LDL = Low Density Lipoprotein; HDL = High Density Lipoprotein; $r=$ spearman correlation coefficient; SBP $=$ Systolic Blood Pressure; WHR = Waist to Hip Ratio

CAC measurements, which is usually done in categories of $\mathrm{CAC}$, again the relations will be valid given our high kappa coefficients. Although our study was performed in healthy postmenopausal women, we expect that the finding will also be applicable for men.

Our findings are important in the light of the wider availability of MDCT in countries compared to EBCT. One reason for that is lower equipment cost. Other advantages of
MDCT over EBCT have been suggested to be less quantum noise, thinner section thickness, and simultaneous acquisition of four sections (with 16-slice or with 64-slice), which is reported to reduce misregistration artifact.

In conclusion, our findings demonstrate that coronary calcium measurements by MDCT are highly reproducible and are not affected by scoring protocols, slice thicknesses and technical factors. 

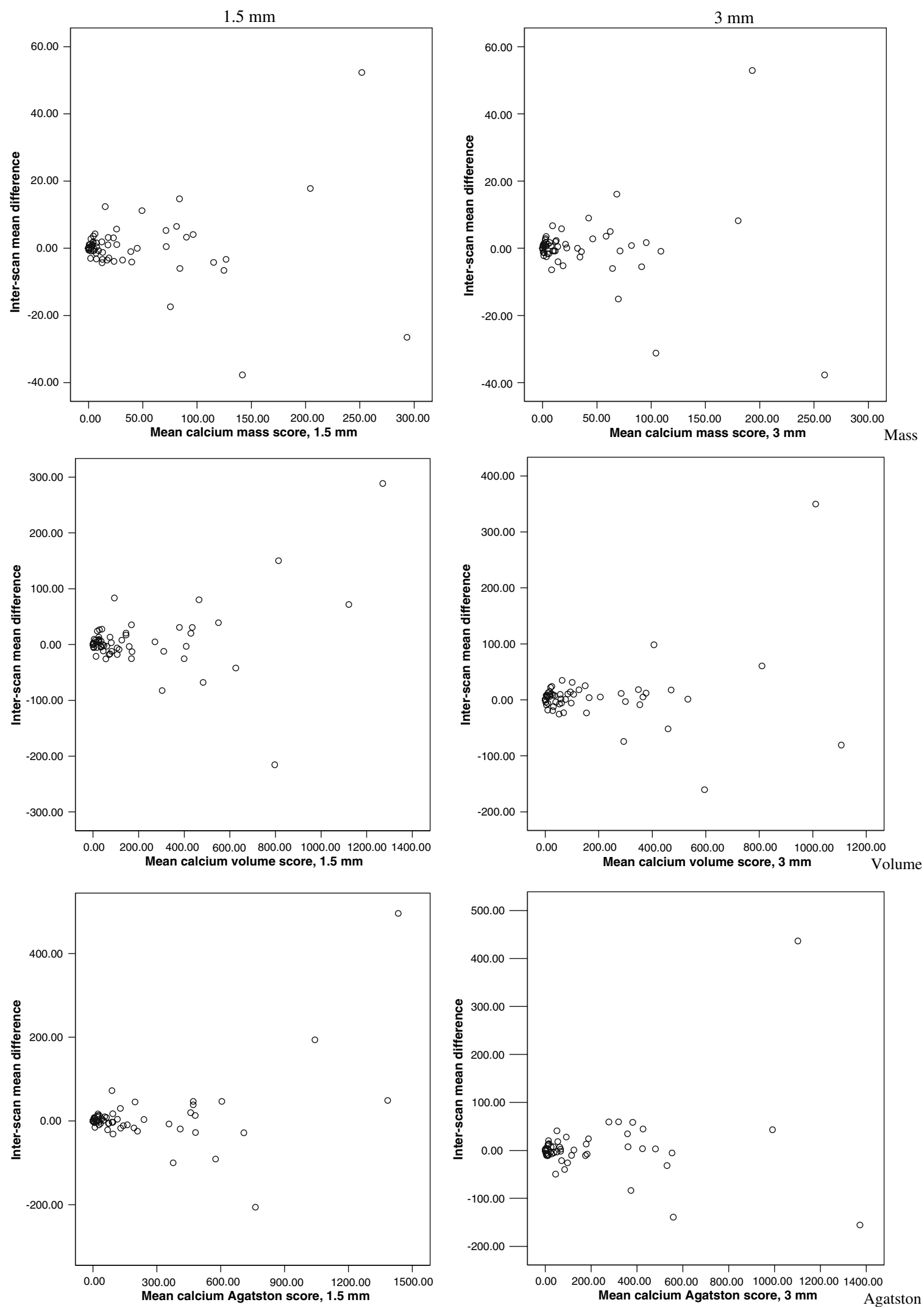

Fig. 1 Relation between mean calcium score and inter-scan difference in mean calcium scores (Bland-Altman plots) 

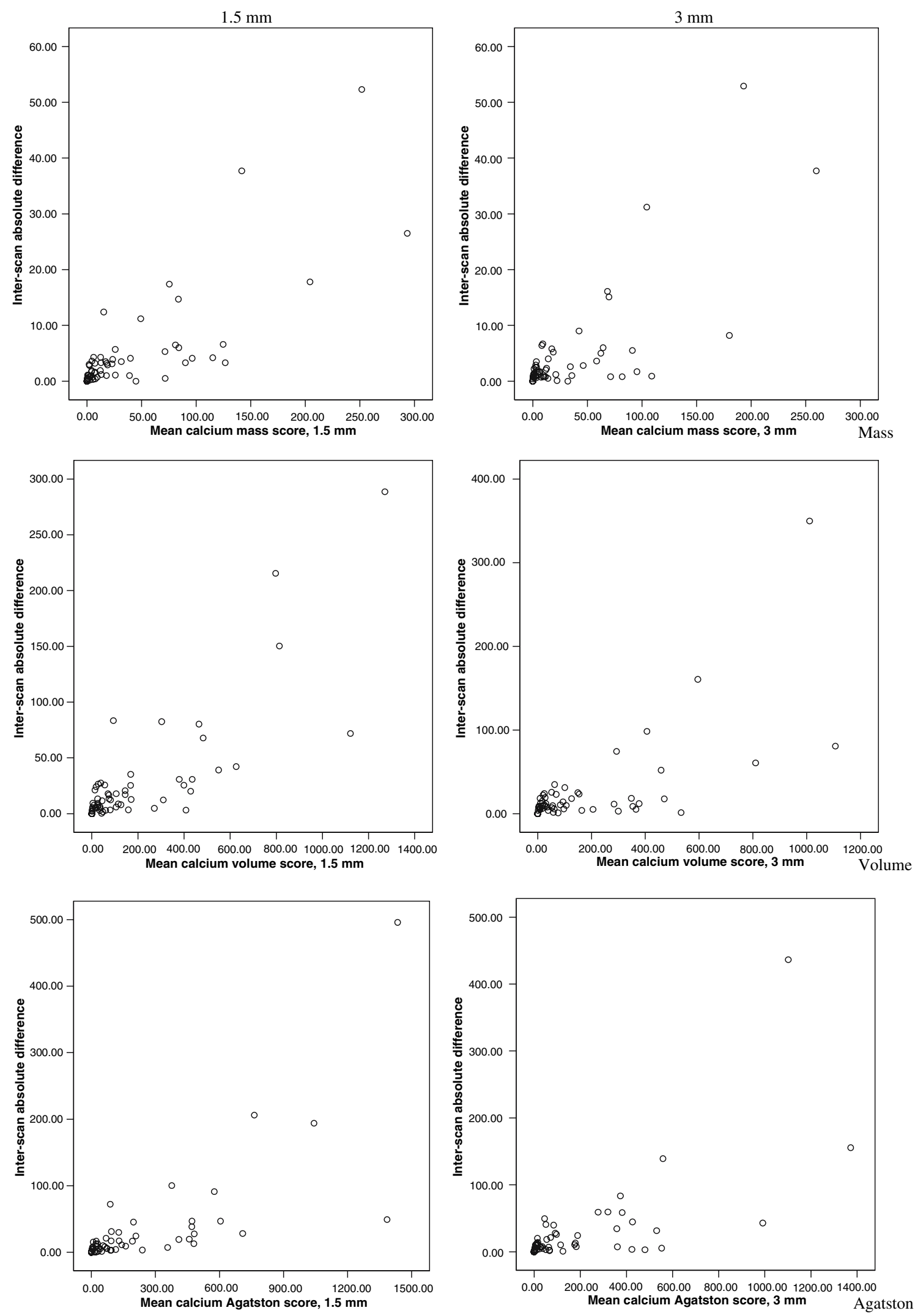

Fig. 2 Relation between mean calcium score and inter-scan absolute difference 
Acknowledgement This study was supported by a grant from The Netherlands Organization for Health Research and Development. First author was supported by Health Ministry of I. R. Iran.

\section{References}

1. Masuda Y, Naito S, Aoyagi Y, Yamada Z, Uda T, Morooka N, et al. Coronary artery calcification detected by CT: clinical significance and angiographic correlates. Angiology 1990;41(12):1037-47

2. Beadenkopf WG, Daoud AS, Love BM. Calcification in the coronary arteries and its relationship to arteriosclerosis and myocardial infarction. Am J Roentgenol Radium Ther Nucl Med 1964;92:865-71

3. Warburton RK, Tampas JP, Soule AB, Taylor HC III. Coronary artery calcification: its relationship to coronary artery stenosis and myocardial infarction. Radiology 1968;91(1):109-15

4. Frink RJ, Achor RW, Brown AL Jr, Kincaid OW, Brandenburg RO. Significance of calcification of the coronary arteries. Am J Cardiol 1970;26(3):241-7

5. Wang S, Detrano RC, Secci A, Tang W, Doherty TM, Puentes G, et al. Detection of coronary calcification with electron-beam computed tomography: evaluation of interexamination reproducibility and comparison of three image-acquisition protocols. Am Heart J 1996;132(3):550-8

6. Nasir K, Budoff MJ, Post WS, Fishman EK, Mahesh M, Lima JA, et al. Electron beam CT versus helical CT scans for assessing coronary calcification: current utility and future directions. Am Heart J 2003;146(6):969-77

7. Thompson BH, Stanford W. Imaging of coronary calcium: a case for electron beam computed tomography. $\mathrm{J}$ Thorac Imaging 2001;16(1):8-15

8. Knez A, Becker C, Becker A, Leber A, White C, Reiser M, et al. Determination of coronary calcium with multi-slice spiral computed tomography: a comparative study with electron-beam CT. Int J Cardiovasc Imaging 2002;18(4):295-303

9. Stanford W, Thompson BH, Burns TL, Heery SD, Burr MC. Coronary artery calcium quantification at multi-detector row helical CT versus electron-beam CT. Radiology 2004;230(2): $397-402$
10. Kopp AF, Ohnesorge B, Becker C, Schroder S, Heuschmid M, Kuttner A, et al. Reproducibility and accuracy of coronary calcium measurements with multi-detector row versus electronbeam CT. Radiology 2002;225(1):113-9

11. Ohnesorge B, Flohr T, Fischbach R, Kopp AF, Knez A, Schroder $\mathrm{S}$, et al. Reproducibility of coronary calcium quantification in repeat examinations with retrospectively ECG-gated multisection spiral CT. Eur Radiol 2002;12(6):1532-40

12. Boker LK, van Noord PA, van der Schouw YT, Koot NV, Bueno de Mesquita HB, Riboli E, et al. Prospect-EPIC Utrecht: study design and characteristics of the cohort population. European Prospective Investigation into Cancer and Nutrition. Eur J Epidemiol 2001;17(11):1047-53

13. Agatston AS, Janowitz WR, Hildner FJ, Zusmer NR, Viamonte M Jr, Detrano R. Quantification of coronary artery calcium using ultrafast computed tomography. J Am Coll Cardiol 1990;15(4): $827-32$

14. Hong C, Bae KT, Pilgram TK. Coronary artery calcium: accuracy and reproducibility of measurements with multi-detector row CT-assessment of effects of different thresholds and quantification methods. Radiology 2003;227(3):795-801

15. Rumberger JA, Brundage BH, Rader DJ, Kondos G. Electron beam computed tomographic coronary calcium scanning: a review and guidelines for use in asymptomatic persons. Mayo Clin Proc 1999;74(3):243-52

16. Reilly MP, Wolfe ML, Localio AR, Rader DJ. Coronary artery calcification and cardiovascular risk factors: impact of the analytic approach. Atherosclerosis 2004;173(1):69-78

17. Rumberger JA, Kaufman L. A rosetta stone for coronary calcium risk stratification: agatston, volume, and mass scores in 11,490 individuals. AJR Am J Roentgenol 2003;181(3):743-8

18. Kaufmann RB, Sheedy PF, Breen JF, Kelzenberg JR, Kruger BL, Schwartz RS, et al. Detection of heart calcification with electron beam CT: interobserver and intraobserver reliability for scoring quantification. Radiology 1994;190(2):347-52

19. Hernigou A, Challande P, Boudeville JC, Sene V, Grataloup C, Plainfosse MC. Reproducibility of coronary calcification detection with electron-beam computed tomography. Eur Radiol 1996;6(2):210-6

20. Bielak LF, Kaufmann RB, Moll PP, McCollough CH, Schwartz RS, Sheedy PF. Small lesions in the heart identified at electron beam CT: calcification or noise? Radiology 1994;192(3):631-6 